\title{
Método Mãe-Canguru: evolução ponderal de recém-nascidos
}

\author{
Kangaroo Mother Method: newborn weight outcome
}

Método Madre Canguro: evolución ponderal de recién nacidos

\section{Juliana de Oliveira Freitas ${ }^{1}$, Climene Laura de Camargo²}

\begin{abstract}
RESUMO
Objetivos: analisar a evolução ponderal de recém-nascido $(\mathrm{RN})$ na $2^{\mathrm{a}}$ etapa do Método Mãe-Canguru e a influência do aleitamento materno no ganho de peso. Métodos: pesquisa quantitativa, prospectiva com $22 \mathrm{RN}$ na $2^{\mathrm{a}}$ etapa do método, entre março e agosto de 2005 . Os dados foram computados no Programa Epi-info. Resultados: 54,6\% estavam abaixo do percentil 5 na alta; as médias das variáveis estudadas foram: tempo para atingir pesos mínimo e de recuperação 5,2 e 17,4 dias; percentual de perda de peso 9,8\%; velocidade relativa de ganho de peso na $1^{\mathrm{a}}$ etapa $9 \mathrm{~g} / \mathrm{Kg} / \mathrm{dia}$ e $2^{\mathrm{a}}$ etapa $13,2 \mathrm{~g} / \mathrm{Kg} /$ dia; velocidade relativa de ganho de peso dos $\mathrm{RN}$ alimentados com leite da genitora 11,9 $\mathrm{g} / \mathrm{Kg} / \mathrm{dia}$ e dos alimentados com leite da genitora e leite pasteurizado $14,5 \mathrm{~g} / \mathrm{Kg} / \mathrm{dia}$. Conclusões: este estudo revela a necessidade de acompanhar RN em pequeno, médio e longo prazo e ampliar as discussões sobre o acompanhamento do crescimento como uma estratégia de atenção básica, visando uma assistência de qualidade.
\end{abstract}

Descritores: Peso-idade; Prematuro; Aleitamento materno

\begin{abstract}
Objectives: To evaluate the pattern of newborns weight gain in the second phase of the Kangaroo Mother Method and to determine the influence of breast feeding on newborns weight gain Methods: This was a prospective study of 22 newborns. Data were collected from March to August 2005. Results: More than a half (54.6\%) of the newborns had weight below the 5 percentile of the weight curve. The percentile of weight loss was of $9.8 \%$. The mean of days for newborns to achieve the minimum weight and progress to normal weight was 5.2 days and 17.4 days, respectively. The relative rate of weight gain was $9 \mathrm{~g} / \mathrm{kg} / \mathrm{day}$ and $13.2 \mathrm{~g} / \mathrm{kg} / \mathrm{day}$ in the first phase and in the second phase, respectively. The relative rate of weight gain of newborns who breast fed at the second phase was of $11.9 \mathrm{~g} / \mathrm{kg} / \mathrm{day}$. And, the relative rate of weight gain of newborns who breast fed and who were also fed with milk from the Human Milk Bank was $14.5 \mathrm{~g} / \mathrm{kg} / \mathrm{day}$. Conclusions: This study suggests the importance of short, medium and long term follow-up of newborns. Monitoring the growth of newborns may be a strategy to improve the quality care to newborns and their families.
\end{abstract}

Keywords: Weight by age; Infant, premature; Breast feeding

\section{RESUMEN}

Objetivo: analizar la evolución ponderal de recién nacidos $(\mathrm{RN})$ en la $2^{\mathrm{a}}$ etapa del Método Madre Canguro y analizar la influencia de la lactancia materna en la ganancia de peso. Métodos: investigación cuantitativa, prospectiva realizada con $22 \mathrm{RN}$ insertados en la $2^{\mathrm{a}}$ etapa del método, entre marzo y agosto del 2005. Los datos fueron computados en el Programa Epi-info. Resultados: El 54,6\% estaban debajo del percentil 5 de la curva de crecimiento al momento del alta; los promedios de las variables estudiadas fueron: tiempo de 5,2 e 17,4 días para alcanzar el peso mínimo y el peso de recuperación; porcentaje de pérdida de peso 9,8\%; velocidad relativa de ganancia de peso en la $1^{\mathrm{a}}$ etapa $9 \mathrm{~g} / \mathrm{Kg} /$ dia y en la $2^{\mathrm{a}}$ etapa $13,2 \mathrm{~g} / \mathrm{Kg} /$ dia; velocidad relativa de ganancia de peso de los $\mathrm{RN}$ alimentados con leche de la progenitora 11,9 $\mathrm{g} / \mathrm{Kg} /$ dia y de los RN alimentados con leche de la progenitora y leche pasteurizada $14,5 \mathrm{~g} / \mathrm{Kg} / \mathrm{dia}$. Conclusiones: este estudio revela la necesidad de acompañar a los RN en pequeño, mediano y largo plazo y ampliar las discusiones sobre el acompañamiento de su crecimiento como una estrategia de atención básica, con miras a brindar atención de calidad a los RN y sus familias.

Descriptores: Peso por edad; Prematuro; Lactancia materna

${ }^{1}$ Enfermeira. Especialista em Enfermagem Neonatal. Mestre em Enfermagem - Escola de Enfermagem da Universidade Federal da Babia - UFBA Salvador (BA), Brasil.

${ }^{2}$ Professora Adjunta da Escola de Enfermagem da Universidade Federal da Babia - UFBA - Salvador (BA), Brasil; Doutora em Saúde Publica pela Faculdade de Saúde Publica da Universidade de São Paulo - USP - São Paulo (SP), Brasil. 


\section{INTRODUÇÃO}

A prematuridade e as condições a ela associadas contribuem com elevados índices de mortalidade nos países em desenvolvimento. Apesar da redução nos índices de mortalidade infantil no Brasil, as taxas de mortalidade perinatal e neonatal vêm aumentando em termos proporcionais, tendo como principais causas o baixo peso e a prematuridade, tornando-se um problema de saúde pública, aumentando custos sociais e econômicos e causando danos no processo de crescimento e desenvolvimento da criança ${ }^{(1)}$.

A monitorização do crescimento pós-natal de recémnascidos ( $\mathrm{RN}$ ) prematuros envolve muitos fatores e deve ser realizada através do controle de peso, do comprimento e do perímetro cefálico. Estes dados devem ser transportados para a curva de crescimento intra-uterino, de acordo com as correspondentes idades pósconcepcionais $^{(2)}$.

O peso constitui uma importante variável de avaliação do crescimento e a evolução ponderal do RN prematuro é fator considerado prioritário em serviços de neonatologia como um dos critérios para a análise do seu crescimento e desenvolvimento, determinando a alta hospitalar.

Antes da idealização do Método Mãe-Canguru MMC, os serviços de atenção neonatal mantinham os prematuros nas incubadoras até alcançarem o peso ideal para alta, o que trazia implicações para a mãe e seu filho, tais como: desestímulo ao aleitamento materno; rompimento do vínculo afetivo; tempo de permanência prolongado nas unidades de internamento, entre outros.

A partir do MMC, padronizado pelo Ministério da Saúde no nosso país em 2002, o RN prematuro e de baixo peso passa a ter contato direto com a mãe, desde o momento em que apresente condições clínicas estáveis. O método encontra-se subdividido em três etapas: na primeira etapa o RN está internado na unidade neonatal, sendo a fase inicial de adaptação ao meio extra-uterino; a segunda etapa corresponde a unidade canguru, e o RN está clinicamente estável e em ganho de peso; por fim, a terceira etapa é o acompanhamento ambulatorial, denominado de follow-up ${ }^{(3)}$.

Nos primeiros dias de internamento em unidades de alto risco o peso dos prematuros sofre influências tanto do seu quadro clínico instável, como dos estressores ambientais aos quais estes RN são submetidos, ocasionando perda de peso característica. Além disso, o contato da criança prematura com os pais se limita ao simples toque, o que torna o estímulo materno e paterno prejudicados. No Método Mãe-Canguru, na medida em que o $\mathrm{RN}$ estabiliza-se clinicamente e inicia-se um processo lento de ganho ponderal, ele passa a ter um contato mais intensificado com sua mãe, em termos de tempo e qualidade. Este contato entre mãe e bebê, proporcionado pelo MMC, estimula o ganho ponderal de forma mais acelerada, a partir do controle da termorregulação e da promoção do aleitamento materno.

Venho atuando no MMC há três anos, e o entendo como uma estratégia de fortalecimento do vínculo materno-neonatal e de reaproximação da equipe de saúde perinatal com o cuidado humanizado. $\mathrm{O}$ interesse pelo presente estudo surgiu da prática profissional com $\mathrm{RN}$ prematuros internados em unidade canguru, o que nos possibilitou vivenciar a contribuição do método no fortalecimento do vínculo afetivo e na redução do tempo de internamento a partir do acelerado ganho de peso, observando, ainda, um crescimento ponderal peculiar entre estes recém-nascidos.

A partir destas discussões, este estudo teve como objetivo geral analisar a evolução ponderal de recémnascidos inseridos na segunda etapa do Método MãeCanguru, a partir do acompanhamento do peso durante a permanência na segunda etapa e, ainda, analisar a influência do aleitamento materno exclusivo no ganho de peso dos recém-nascidos estudados.

\section{MÉTODOS}

Trata-se de um estudo quantitativo, descritivo, de seguimento prospectivo, realizado na Unidade Canguru de uma maternidade pública do município de Feira de Santana. A população foi composta por todos os RN inseridos na segunda etapa do MMC no período de março a agosto de 2005, totalizando 22 bebês. A segunda etapa foi escolhida porque os RN encontram-se estáveis clinicamente, em ganho de peso, possibilitando melhor avaliação ponderal, além de evitar as possíveis perdas por óbitos na $1^{\text {a }}$ etapa, ou impossibilidade de pesagem devido à gravidade.

Como instrumento de coleta de dados foi utilizado um formulário, preenchido a partir da entrevista estruturada com as puérperas; da pesagem semanal dos RN e da investigação documental através do prontuário.

O projeto foi apreciado e aprovado pelo Comitê de Ética em Pesquisa da Universidade Estadual de Feira de Santana.

A monitorização do peso pós-natal foi realizada através do controle semanal em balança digital com capacidade de pesagem $15 \mathrm{Kg}$ x $5 \mathrm{~g}$ que atende a Norma do Instituto Nacional de Metrologia - INMETRO. Foram colhidos do prontuário os dados de peso ao nascer, peso mínimo atingido, peso de recuperação (primeiro peso igual ou superior ao peso de nascimento) e peso de admissão na $2^{a}$ etapa do MMC. Os dados foram transportados para a curva de crescimento ponderal construída a partir de uma tese de doutorado apresentada a Faculdade de Medicina de Ribeirão Preto 
- São Paulo ${ }^{(4)}$. Nesta, são propostas duas curvas de acompanhamento do peso do RNPT: uma para o sexo masculino e outra para o feminino, estabelecendo os percentis 5 , 50 e $90^{(5)}$.

Nesta pesquisa, além do acompanhamento pelas curvas de crescimento, foram calculadas a porcentagem de perda de peso, as taxas de velocidade absoluta e relativa de perda de peso, as taxas de velocidade absoluta e relativa de ganho de peso, as taxas de velocidade absoluta e relativa de ganho de peso na $1^{\mathrm{a}}$ e $2^{\mathrm{a}}$ etapas do MMC e as taxas de velocidade relativa de ganho de peso dos $\mathrm{RN}$, segundo o tipo de leite utilizado na $2^{\mathrm{a}}$ etapa do MMC.

Os dados foram computados no banco construído no Programa Epi Info 6.04d. Foram realizadas análises de freqüências simples a partir dos resultados obtidos, e estes estão apresentados sob a forma de tabelas de contingência.

\section{RESULTADOS}

Durante o período deste estudo o número de nascidos vivos na instituição foi de 2.527. Destes, 235 foram internados no Berçário de Risco (1 $1^{a}$ etapa do MMC), dos quais $107(44,6 \%)$ eram prematuros ou apresentaram baixo peso de nascimento, e $22(20,5 \%)$ participaram da $2^{a}$ etapa do MMC, grupo este que constituiu a população deste estudo.

Tabela 1 - Freqüência do peso dos recém-nascidos inseridos na segunda etapa do segundo percentil de crescimento. Maringá, março a agosto de 2005.

\begin{tabular}{lrr}
\hline PESO & N & \% \\
\hline Menor igual Percentil 5 & 12 & 54,6 \\
Percentil 5 - percentil 50 & 7 & 31,8 \\
Maior igual Percentil 50 & 3 & 13,6 \\
\hline TOTAL & $\mathbf{2 2}$ & $\mathbf{1 0 0 , 0}$
\end{tabular}

A Tabela 1 mostra que, 54,6\% daqueles inseridos na $2^{\text {a }}$ etapa do MMC se encontravam abaixo do percentil 5 da Curva de Crescimento, proposta por Xavier, até o momento da alta hospitalar, e 31,8\% entre o percentil 5 e o percentil 50.

\section{Tempo de internamento}

A média de permanência hospitalar calculada entre a $1^{a}$ e $2^{a}$ etapas foi de 29,6 dias de internamento até a alta hospitalar.

\section{Tempo para atingir peso mínimo e peso de recuperação}

O cálculo da média de tempo em que os recémnascidos alcançaram seu peso mínimo durante o internamento foi de 5,2 dias. Já a média de tempo para atingir o peso de recuperação foi de 17,4 dias.

\section{Percentual de perda de peso}

A média do percentual de perda de peso foi de 9,8\%.

Velocidades absoluta e relativa de perda e ganho de peso

As médias das velocidades absoluta e relativa de perda e ganho de peso foi de 27,8 g/dia, $20 \mathrm{~g} / \mathrm{Kg} / \mathrm{dia}, 13,2 \mathrm{~g} /$ dia e $10,8 \mathrm{~g} / \mathrm{Kg} / \mathrm{dia}$, respectivamente.

Velocidades absoluta e relativa de ganho de peso na $1^{a}$ e $2^{a}$ etapas do MMC

$\mathrm{Na} 1^{a}$ etapa do MMC, os RN apresentaram uma média da velocidade absoluta de ganho de peso de 11,2 $\mathrm{g} /$ dia, enquanto a velocidade relativa de ganho de peso foi de $9 \mathrm{~g} / \mathrm{Kg} /$ dia. $\mathrm{Na} 2^{\mathrm{a}}$ etapa do MMC, o cálculo da média da velocidade absoluta de ganho de peso foi de $16,9 \mathrm{~g} /$ dia e da velocidade relativa foi de $13,2 \mathrm{~g} / \mathrm{Kg} / \mathrm{dia}$.

Velocidade relativa de ganho de peso na $2^{\mathrm{a}}$ etapa do MMC segundo tipo de leite utilizado

O cálculo da média da velocidade relativa de ganho de peso dos $\mathrm{RN}$ alimentados com leite da genitora, na $2^{\mathrm{a}}$ etapa, foi de $11,9 \mathrm{~g} / \mathrm{Kg} /$ dia e dos alimentados com leite da genitora, complementado com leite no pasteurizado, foi de $14,5 \mathrm{~g} / \mathrm{Kg} / \mathrm{dia}$.

Tabela 2 - Média das características dos recém-nascidos inseridos na segunda etapa do Método Mãe-Canguru. Maringá, março a agosto de 2005.

\begin{tabular}{|c|c|}
\hline CARACTERÍSTICAS & MÉDIA \\
\hline $\begin{array}{l}\text { Tempo de internamento entre a primeira e } \\
\text { segunda etapas (dias) }\end{array}$ & 29,6 \\
\hline Tempo para atingir peso mínimo (dias) & 5,2 \\
\hline $\begin{array}{l}\text { Tempo para atingir peso de recuperação } \\
\text { (dias) }\end{array}$ & 17,4 \\
\hline Percentual de perda de peso $(\%)$ & 9,8 \\
\hline Velocidade absoluta de perda de peso (g/dia) & 27,8 \\
\hline $\begin{array}{l}\text { Velocidade relativa de perda de peso } \\
(\mathrm{g} / \mathrm{Kg} / \mathrm{dia})\end{array}$ & 20,0 \\
\hline Velocidade absoluta de ganho de peso (g/dia) & 13,2 \\
\hline $\begin{array}{l}\text { Velocidade relativa de ganho de peso } \\
\mathrm{g} / \mathrm{Kg} / \mathrm{dia}\end{array}$ & 10,8 \\
\hline $\begin{array}{l}\text { Velocidade absoluta de ganho de peso na } 1^{\mathrm{a}} \\
\text { etapa (g/dia) }\end{array}$ & 11,2 \\
\hline $\begin{array}{l}\text { Velocidade relativa de ganho de peso naa } 1^{\text {a }} \\
\text { etapa }(\mathrm{g} / \mathrm{Kg} / \text { dia })\end{array}$ & 9,0 \\
\hline $\begin{array}{l}\text { Velocidade absoluta de ganho de peso na } 2^{\mathrm{a}} \\
\text { etapa }(\mathrm{g} / \mathrm{dia})\end{array}$ & 16,9 \\
\hline $\begin{array}{l}\text { Velocidade relativa de ganho de peso na } 2^{\mathrm{a}} \\
\text { etapa }(\mathrm{g} / \mathrm{Kg} / \mathrm{dia})\end{array}$ & 13,2 \\
\hline $\begin{array}{l}\text { Velocidade relativa de ganho de peso dos } \mathrm{RN} \\
\text { alimentados com leite da genitora na } 2^{\mathrm{a}} \text { etapa } \\
\mathrm{g} / \mathrm{Kg} / \mathrm{dia}\end{array}$ & 11,9 \\
\hline $\begin{array}{l}\text { Velocidade relativa de ganho de peso dos } \mathrm{RN} \\
\text { alimentados com leite da genitora } \\
\text { complementado com leite pasteurizado na } 2^{\mathrm{a}} \\
\text { etapa } \mathrm{g} / \mathrm{Kg} / \mathrm{dia}\end{array}$ & 14,5 \\
\hline
\end{tabular}

Quanto ao aleitamento materno exclusivo - AME na alta hospitalar da $2^{\mathrm{a}}$ etapa do MMC, $68,2 \%$ dos RN foram 
para casa mamando exclusivamente no peito da mãe.

Tabela 3 - Distribuição da freqüência dos recém-nascidos inseridos na segunda etapa do Método Mãe-Canguru segundo o aleitamento materno exclusivo na alta. Maringá, março a agosto de 2005.

\begin{tabular}{lrr}
\hline $\begin{array}{l}\text { ALEITAMENTO EXCLUSIVO } \\
\text { NA ALTA }\end{array}$ & $\mathbf{N}$ & $\mathbf{\%}$ \\
\hline Sim & 15 & 68,2 \\
Não & 07 & 31,8 \\
\hline TOTAL & $\mathbf{2 2}$ & $\mathbf{1 0 0 , 0}$ \\
\hline
\end{tabular}

\section{DISCUSSÃO}

De acordo ao percentil de crescimento dos RN estudados, os dados da Tabela 1 reforçam a necessidade do acompanhamento na $3^{a}$ etapa do MMC, já que, no momento da alta hospitalar, a maior parte dos $\mathrm{RN}$ se encontrava abaixo dos percentis adequados (5 a 50) da curva de crescimento adotada. Vale ressaltar que, a maioria das mães $(68,2 \%)$ referiu ser de outro município e não retorna para o hospital de origem, o que dificulta o acompanhamento ambulatorial. Diante disto, seria interessante que os programas de atenção básica pudessem realizar o acompanhamento médico e de enfermagem de crianças nascidas prematuras, referenciando outras especialidades necessárias. Entretanto, para que tal acompanhamento aconteça, seria necessária a adoção de determinadas condutas na atenção básica, tendo como premissa a implantação do cartão da criança prematura.

Quanto ao tempo de internamento estudos revelam resultados semelhantes, ou com tempo de internamento mais prolongado. Estudo que avaliou o crescimento de $141 \mathrm{RN}$ prematuros AIG, descreve uma permanência no berçário relativamente prolongada, já que 35,4\% tiveram alta hospitalar com mais de quatro semanas de vida e $11,3 \%$ com mais de oito semanas de vida pósnatal ${ }^{(4)}$.

Estudo recente que avaliou os fatores associados ao estado nutricional na idade corrigida de termo em RN prematuros com MBP, e a média de dias de internação entre os RN eutróficos e desnutridos foi de 43,2 e 56,5 dias, respectivamente ${ }^{(6)}$.

O Programa Mãe Canguru do Instituto Materno Infantil de Pernambuco (IMIP) descreve como tempo médio de permanência hospitalar 16 dias, sendo bem reduzido quando comparado ao tempo de internação de 60 a 90 dias observado no berçário convencional do IMIP e de outros hospitais de Pernambuco ${ }^{(7)}$.

O tempo de internamento de $\mathrm{RN}$ prematuros e de baixo peso nas unidades hospitalares normalmente é prolongado devido a sua dificuldade de adaptação ao meio extra-uterino. Sabe-se que um maior tempo de internamento pode trazer repercussões no desenvolvimento do $\mathrm{RN}$, levando muitos serviços a buscarem reduzir a estada destas crianças nas unidades hospitalares. Um das estratégias para redução do tempo de internamento hospitalar de recém-nascidos prematuros e de baixo peso é o MMC.

Em relação ao tempo para atingir peso mínimo e peso de recuperação, os recém-nascidos, em geral, apresentam uma perda inicial de peso, seguida da sua recuperação. A literatura corrobora com os dados deste estudo quando traz que o tempo para o recém-nascido prematuro atingir seu peso mínimo é menor que o tempo para recuperar o peso de nascimento, e normalmente eles perdem $10 \%$ do peso ao nascer e ganham em média 20 a $30 \mathrm{~g} / \mathrm{dia}^{(8)}$.

Estudo sobre crescimento de RN pré-termo nas primeiras 12 semanas de vida mostrou que, a perda de peso variou de $5,9 \%$ a $9,7 \%$ do peso de nascimento, ocorrendo dentro da primeira semana de vida, de 4 a 6 dias. Neste mesmo estudo, o peso de recuperação foi atingido entre 16 e 19 dias. Os autores reforçam, ainda, que uma vez recuperado o peso de nascimento, a partir da terceira semana os $\mathrm{RN}$ mantiveram taxas crescentes de ganho de peso $^{(9)}$.

Outro estudo encontrou um tempo para atingir o peso mínimo de 5 dias, e tempo para atingir o peso de recuperação variou de 11 a 21 dias, de acordo a idade gestacional e o peso de nascimento ${ }^{(8)}$. Em pesquisa realizada em 2005 foi detectado que a média de tempo para recuperação do peso de nascimento foi de 16,9 dias para os RN eutróficos e 19,8 dias para os desnutridos $^{(6)}$.

Diante das discussões dos autores anteriormente citados, é possível refletir sobre a necessidade de uma atenção médica e de enfermagem direcionada para melhores práticas nutricionais com o $\mathrm{RN}$ prematuro. Assim, cabe a equipe médica avaliar e indicar precocemente a melhor nutrição para estes $\mathrm{RN}$, e à enfermagem compete o cuidado individualizado, observando e sinalizando as respostas do $\mathrm{RN}$ à nutrição estabelecida, para que sejam feitos ajustes necessários visando um trabalho interdisciplinar ${ }^{(5)}$.

O percentual de perda de peso inicial dos $\mathrm{RN}$ prétermo, é descrito em vários estudos sobre crescimento de prematuros. Artigo de revisão sobre follow-up de risco descreve que uma perda inicial é esperada em todos os RN, sendo chamada nos prematuros de fase de retardo de crescimento, e é maior quanto menor for a idade gestacional, o peso de nascimento e a severidade da doença neonatal ${ }^{(10)}$.

Alguns pesquisadores agruparam RN com MBP de acordo ao peso de nascimento e observaram que, o 
percentual de perda de peso foi de $14,5 \% \pm 3$ entre os $\mathrm{RN}$ menores que $1000 \mathrm{~g}, 14,5 \% \pm 5,7$ naqueles com peso entre 1000 e $1249 \mathrm{~g}$, e 9,3\% \pm 4,1 pra os RN que pesavam entre 1250 e $1499 \mathrm{~g}^{(8)}$.

Pesquisa de 2004 descreve que, como resultado da contração do volume extracelular e do catabolismo, o RN perde de 5 a $10 \%$ do seu peso ao nascer. Essa perda é inversamente proporcional ao peso de nascimento e os $\mathrm{RN}$ com MBP podem perder de 10 a $20 \%$, ou seja, 2 a $3 \%$ do seu peso por $\mathrm{dia}^{(11)}$.

Assim, os RN prematuros e com muito baixo peso sofrem uma perda de peso inicial maior em relação aos $\mathrm{RN}$ a termo e com peso adequado. Após atingir seu peso mínimo, os pré-termo alcançam e recuperam o peso ao nascer com tendência a um aumento acelerado deste peso, mediante condições clínicas e nutricionais adequadas.

No que se refere às velocidades absoluta e relativa de perda e ganho de peso, para avaliação do crescimento ponderal dos $\mathrm{RN}$ prematuros, os profissionais de saúde podem utilizar taxas absolutas calculadas em $\mathrm{g} / \mathrm{dia}$ ou taxas relativas calculadas em $\mathrm{g} /$ $\mathrm{kg} /$ dia. Principalmente em relação ao peso, as taxas absolutas ( $\mathrm{g} / \mathrm{dia}$ ) podem não ser adequadas. No entanto, observa-se que as taxas relativas $(\mathrm{g} / \mathrm{Kg} / \mathrm{dia})$ mostram um crescimento adequado ${ }^{(12)}$. Neste estudo, quando se calcula a taxa absoluta, observa-se que, os RN tendem a perder mais peso em relação à taxa relativa. Já quanto ao ganho, os dados mostraram que os percentuais são semelhantes e quando se calcula a velocidade absoluta de ganho de peso, os RN tendem a ganhar mais peso em relação à velocidade relativa.

Estudo sobre a velocidade de crescimento de prematuros AIG, chamam a atenção para a importância de que as taxas de crescimento sejam alcançadas a todo o momento e que possam ser aceleradas ou diminuídas de acordo com essa avaliação ${ }^{(12)}$.

As médias das velocidades absoluta e relativa variaram e aumentaram da $1^{a}$ para a $2^{a}$ etapa do MMC. Podemos destacar que o ganho de peso na $2^{\text {a }}$ etapa foi consideravelmente maior que na $1^{a}$ etapa, independente da taxa de velocidade calculada.

O ganho de peso maior na $2^{\mathrm{a}}$ etapa pode ser associado ao processo de adaptação às injúrias sofridas pelo $\mathrm{RN}$ na unidade de risco ( $1^{\text {a }}$ etapa do $\mathrm{MMC}$ ) e, ainda, à descontinuidade do contato e do estímulo materno.

O MMC do Instituto Materno Infantil de Bogotá, entre os anos de 1979 e 1986, realizou o atendimento de 1654 prematuros com peso menor que $2000 \mathrm{~g}$ e o ganho ponderal no primeiro ano de vida superou em quatro vezes e meio o peso de nascimento ${ }^{(13)}$.

Uma revisão de literatura sobre a recuperação do $\mathrm{RN}$ em UTI neonatal, mostra que o MMC não substitui as UTI, mas o método supre as necessidades do RN, levando ao melhor desenvolvimento, propiciando o incentivo ao aleitamento materno, o calor da mãe, carícias, enfim, influências que contribuem na sua recuperação, condições estas não viabilizadas pelos equipamentos de uma UTI ${ }^{(14)}$.

Diante das afirmações dos autores citados, é importante destacar que os benefícios do MMC estão além do ganho de peso mais acelerado, pois promovem fortalecimento da relação mãe e filho, minimizando o estresse físico e psicológico sofrido por ambos.

Quanto a velocidade relativa de ganho de peso na $2^{\mathrm{a}}$ etapa do MMC segundo tipo de leite utilizado, observamos que os RN alimentados exclusivamente com leite materno de sua genitora apresentaram uma velocidade relativa de ganho de peso menor que os $\mathrm{RN}$ alimentados com leite da mãe complementado com leite humano pasteurizado pelo Banco de Leite Humano.

Estudos que utilizaram leite posterior da própria mãe, mais rico em gordura, resultou num melhor ganho de peso entre os RN prematuros ${ }^{(15)}$. Assim, o uso do leite posterior poderia ser uma estratégia utilizada naqueles que não estão alcançando o peso esperado, a fim de priorizar o uso do leite materno exclusivo nos pré-termos.

Avalição sobre o crescimento de RN pré-termo de 1200 a 2000 gramas de peso ao nascers acompanhados na metodologia mãe-canguru, mostrou que é possível identificar aqueles que crescem exclusivamente com leite materno e aqueles que necessitaram de suplementação temporária ou prolongada, para proteger seu potencial de crescimento $^{(9-12)}$.

Artigo sobre enriquecimento do leite humano descreve que numerosos estudos documentam que o leite humano é melhor do que a fórmula para lactentes prematuros, tanto para o crescimento imediato como para o desenvolvimento a longo prazo. Em contrapartida, o leite humano prematuro e o leite humano maduro coletado não suprem as quantidades adequadas de diversos nutrientes para preencher as necessidades nutricionais do lactente prematuro, particularmente daqueles com muito baixo peso ${ }^{(16)}$.

Discussão sobre os benefícios clínicos do leite humano para o lactente prematuro coloca que a alimentação exclusiva destes com leite humano não enriquecido, em especial nos menores que $1500 \mathrm{~g}$, tem resultado em taxas de crescimento insuficientes, associadas a carências nutricionais durante e além do período de internamento hospitalar ${ }^{(17)}$.

O leite produzido pela mãe do $\mathrm{RN}$ prematuro é considerado o melhor alimento para estes bebês, sendo importante, principalmente, em países em desenvolvimento devido os custos dos substitutos do leite humano. Os RN têm crescimento adequado com leite da própria mãe, porém pode ser preciso adaptá-lo para crianças menores de 1500 gramas que necessitam de quantidades maiores de proteína, sódio, cálcio e fósforo, complementos ou fortificantes do leite humano ${ }^{(18)}$. 
Torna-se necessário estabelecer metas mais apropriadas que incluam, não só o crescimento compensatório ou crescimento em catch-up, mas que levem em consideração os benefícios do uso do leite humano para a prevenção da morbidade relacionada à alimentação e otimização dos resultados em longo prazo, sob os aspectos do desenvolvimento do sistema neural ${ }^{(19)}$.

Assim, a decisão quanto a alimentar o $\mathrm{RN}$ prematuro com leite materno exclusivo, com leite humano suplementado ou, ainda, com fórmulas para pré-termos, a fim de suprir suas necessidades nutricionais e de crescimento ainda permanece sob discussão.

Quanto ao aleitamento materno exclusivo - AME no momento da alta hospitalar, a maioria dos $\mathrm{RN}$ foram para casa mamando exclusivamente no peito da mãe. Poderíamos atribuir tal resultado ao fato da instituição estudada ter o título de Hospital Amigo da Criança, desenvolvendo um programa de incentivo ao aleitamento materno, e ainda ao MMC.

Um estudo realizado em 2003 sobre o desenvolvimento de $\mathrm{RN}$ inseridos no MMC mostrou, aos seis meses de vida, uma prevalência de $60,3 \%$ de AME entre as crianças que participaram do programa ${ }^{(20)}$.

O programa mãe canguru do IMIP, traz como um dos principais resultados o maior tempo de aleitamento materno. Cerca de $95 \%$ dos $\mathrm{RN}$ do programa receberam alta em AME e $30 \%$ chegaram ao $6^{\circ}$ mês de vida mamando exclusivamente no peito da mãe $e^{(6)}$.

Numa revisão sobre manejo clínico hospitalar do aleitamento materno em $\mathrm{RN}$ prematuros, para o prétermo as recomendações do aleitamento materno têm sido defendidas com base nas propriedades imunológicas, no seu papel na maturação gastrintestinal, na formação do vínculo mãe e filho e no melhor desempenho neurocomportamental apresentado pelas crianças amamentadas. Além disso, os autores afirmam que o alimento de escolha para o RN prematuro é o leite da própria mãe $\mathrm{e}^{(15)}$.

Os autores descritos reforçam a necessidade de estratégias para o incentivo ao aleitamento materno entre os pré-termo, destacando a sua influência positiva no crescimento e desenvolvimento da criança nascida prematuramente.

\section{CONCLUSÃO}

O cuidado a $\mathrm{RN}$ prematuros vem sendo amplamente discutido sob vários aspectos, com o objetivo de promover melhor qualidade de vida para estas crianças e suas famílias e melhores condições para o crescimento e desenvolvimento saudáveis, já que, devido aos investimentos e avanços na atenção neonatal, sua sobrevida aumentou sensivelmente.

Os prematuros constituem uma população que obedece a um perfil de crescimento muito peculiar, podendo vir a sofrer influência de determinados fatores relacionados à maturidade, às condições clínicas e ao suporte nutricional que lhes é oferecido.

Para acompanhar o crescimento de crianças nascidas prematuras torna-se necessário lançar mão de curvas de crescimento adequadas para esta clientela, as quais consideram a idade gestacional corrigida, e não a idade cronológica.

Diante dos resultados obtidos, análises e discussões realizadas nesta pesquisa, foi possível conhecer a dinâmica de crescimento ponderal da população estudada, possibilitando possíveis intervenções assistenciais voltadas para a promoção de um ganho ponderal mais adequado ao RN prematuro ou com baixo peso. Podemos afirmar que o crescimento ponderal destes $\mathrm{RN}$ necessita de uma avaliação individualizada, para melhor adequação nutricional e acompanhamento após alta hospitalar.

Ressaltamos a necessidade de promover a difusão de programas voltados para a população de crianças nascidas prematuras e com baixo peso, a exemplo do Programa Mãe-Canguru. Este visa o fortalecimento de estratégias que promovem a participação da família no cuidado do seu filho, e ainda incentiva a promoção do aleitamento materno, tão importante para o crescimento e o desenvolvimento saudáveis desta população.

Este estudo aponta a necessidade de acompanhar esses $\mathrm{RN}$ em pequeno, médio e longo prazo. É preciso discutir sobre o acompanhamento do crescimento e do desenvolvimento dos prematuros como uma estratégia de atenção básica em saúde, e não apenas, em serviços especializados de assistência neonatal, dos quais o País é ainda muito carente.

Destacamos que este trabalho de pesquisa foi realizado com uma pequena população de $\mathrm{RN}$ prematuros de um serviço específico, o que lhe traz limitações, dificultando generalizações e sugerindo a realização de estudos mais amplos. Entretanto, foi possível trazer contribuições para o serviço, no sentido de conhecer a população estudada sob os aspectos do seu crescimento ponderal, promovendo mudanças no manejo nutricional destes bebês.

Resgatamos a afirmação de que cuidar de crianças nascidas prematuras é um desafio e, desta forma, os profissionais que atuam na assistência neonatal necessitam de constante aprimoramento e apoio de órgãos gestores em saúde, para que possamos proporcionar uma atenção de qualidade aos nossos pequeninos e respectivas famílias.

\section{REFERENCIAS}

1. Gaíva MAM, Gomes MMF. Cuidando do neonato: uma abordagem de enfermagem. Goiânia: AB; 2003.

2. Leone CR, Tronchin DMR. Assistência integrada ao recém- 
nascido. São Paulo: Atheneu; 1996.

3. Brasil. Ministério da Saúde. Secretaria de Políticas de Saúde. Atenção humanizada ao recém-nascido de baixo peso, método mãe canguru. Brasília: Ministério da Saúde; 2002.

4. Xavier CC, Abdallah VOS, Silva BR, Mucillo G, Jorge SM, Barbieri MA. Crescimento de recém-nascido pré-termo. J Pediatr (Rio de J).1995; 71(1):22-7.

5. Xavier CC, Anchieta LM, Ornelas SL. Crescimento do recémnascido pré-termo. Temas de Pediatria. 2004; (77): 3-24.

6. Gianini NM, Vieira AA, Moreira MEL. Avaliação dos fatores associados ao estado nutricional na idade corrigida de termo em recém-nascidos de muito baixo peso. J Pediatr (Rio de J). 2005; 81(1):34-40.

7. Guimarães MCL. O programa mãe-canguru do Instituto Materno Infantil de Pernambuco. In: Fujiwara LM, Alessio NLN, Farah MFS, organizadores. 20 experiências de gestão pública e cidadania. São Paulo: Programa Gestão Pública e Cidadania; 1998. p. 3-11.

8. Uliani ACCA, Carvalho R, Barros Filho AA. Evolução ponderal de recém-nascidos de muito baixo peso. J Pediatr (Rio de J). 1996; 72(6):388-93.

9. Anchieta LM, Xavier CC, Colosimo EA. Crescimento de recém-nascidos pré-termo nas primeiras 12 semanas de vida. J Pediatr (Rio de J). 2004; 80(4):267-76.

10. Porto MACSC. Como avaliar o crescimento de prematuros. In: Rio de Janeiro. Secretaria Municipal de Saúde, organizador. Manual de acompanhamento clínico do recém-nascido de risco. Rio de Janeiro: Oficinas Gráficas da Imprensa da Cidade do Rio de Janeiro; 2000.

11. Espiridião S, Costa HPF, Zucolotto FB. Terapia hidroletrolítica. In: Costa HPF, Marba ST. O recém-nascido de muito baixo peso. São Paulo: Atheneu; 2004. p.121-48.

12. Anchieta LM, Xavier CC, Colosimo EA. Velocidade de crescimento de recém-nascidos pré-termo adequados para a idade gestacional. J Pediatr (Rio de J). 2004; 80(5):417-24.

13. Charpak N, Calume ZF, Hamel A. O método mãe-canguru. Rio de Janeiro: McGraw-Hill; 1999.

14. Ferreira L, Viera CS. A influência do método mãe-canguru na recuperação do recém-nascido em Unidade de Terapia Intensiva Neonatal: uma revisão de literatura. Acta Sci, Health Sci. 2003; 25(1):41-50.

15. Nascimento MBR, Issler H. Aleitamento materno em prematuros: manejo clínico hospitalar. J Pediatr (Rio de J). 2004; 80(5 Supl):S163-S172.

16. Moro GE, Minoli I. Enriquecimento do leite humano [resumo]. In: o $43^{\circ}$ Seminário Nestlé Nutricion. 1998. p. 18-9.

17. Schanler RJ. Benefícios clínicos do leite humano par o lactente prematuro [resumo]. In: $43^{\circ}$ Seminário Nestlé Nutricion. 1998. p.20-3.

18. Tavares EC, Xavier CC, Lamounier JA. Nutrição e alimentação do recém-nascido prematuro. Rev Med Minas Gerais. 2003; 13(4 Supl 2):S54-9.

19. Toma TS. Método mãe-canguru: alimentação do recémnascido prematuro e/ou de baixo peso [texto na Internet]. 2003. [citado 2005 Mai 9]. Disponível em: http:// www.aleitamento.com.

20. Venancio SI, Almeida H. Método Mãe Canguru: aplicação no Brasil, evidências científicas e impacto sobre o aleitamento materno. J Pediatr (Rio de J). 2004; 80(5 Supl):S173- S180. 